\title{
Chromatography-Free Esterification Reactions Using a Bifunctional Polymer
}

\author{
Shuang Ma, Patrick H. Toy ${ }^{*}$ \\ Department of Chemistry, The University of Hong Kong, Pokfulam Road, Hong Kong, P. R. of China \\ Fax +852 28571586; E-mail: phtoy@hku.hk
} Abstract: A linear polystyrene functionalized with both nucleo-
philic DMAP groups and sterically hindered tertiary amine groups
was synthesized and used homogeneously in a range of esterifica-
tion reactions between alcohols and various carboxylic acid deriv-
atives. The polymer was highly effective in such reactions where
the DMAP groups served as catalytic groups. The ester products
of these reactions could be isolated in high purity and yield with-
out the need for chromatographic purification, and the polymer
could be recovered and reused numerous times with no apparent
decrease in utility.

Key words: DMAP, esterification reactions, organocatalysis, polymer-supported catalysts, polymer-supported reagents

4-Dimethylaminopyridine (DMAP) is a versatile nucleophilic organocatalyst that is perhaps most commonly used to catalyze ester forming reactions between an alcohol and a carboxylic acid derivative. ${ }^{1}$ Due to its versatility, many polymer-supported versions of DMAP have been prepared with the aim of facilitating product isolation, and for enabling catalyst recycling. when DMAP is used in an ester forming reaction, including supported versions, an auxiliary sterically hindered tertiary amine base is added to neutralize the acid byproduct that is formed. In this area of research, we have previously reported rasta resin-supported DMAP (1, Figure 1) as a catalyst for reactions between epoxides and carbon dioxide to form cyclic carbonates. ${ }^{4}$ We have also prepared bifunctional polystyrenes $2^{5}$ and $3^{6}$ that were effective catalysts for Morita-Baylis-Hillman and decarboxylative Doebner-Knoevenagel reactions, respectively. Both functional groups of polymers $\mathbf{2}$ and $\mathbf{3}$ were found to be required for effective catalysis of the reactions studied, and we wanted to extend our research by developing a polystyrene-supported DMAP catalyst that also possessed auxiliary tertiary amine base reagent groups for use in ester forming reactions that did not require the need for chromatographic purification of the desired product. ${ }^{7}$ Herein we report the realization of this objective through the synthesis and use of a bifunctional polystyrene.

As in all of our prior research regarding multifunctional polymers, we used the notion of simple monomer synthesis in 1 or 2 steps from commercially available starting materials as a guiding principle in order to make our polymer as inexpensive and practical as possible. ${ }^{8}$ Thus, monomers $4^{4}$ and $5^{8 \mathrm{c}}$ were prepared according to our previously reported procedures from 4-vinyl benzyl chloride and the appropriate amine using substitution reactions. Monomers 4 and 5 were heated in a 1:6 ratio in toluene at $85{ }^{\circ} \mathrm{C}$ for 36 hours to afford polymer $\mathbf{6}$ in $58 \%$ yield as a free-flowing powder after precipitation from $\mathrm{MeOH}$ (Scheme 1). 9 This monomer ratio was chosen because we wanted $\mathbf{6}$ to have at least 5 tertiary amine groups for each DMAP group since we anticipated using at most 20 mole percent of the catalytic groups in the esterification reactions. ${ }^{1} \mathrm{H}$ NMR analysis was used to determine the ratio of monomer incorporation in $\mathbf{6}$, and indicated that it possessed essentially the same 1:6 ratio of DMAP to tertiary amine groups as the ratio of starting materials used in the polymerization reaction. This ratio corresponds to a DMAP loading level of $0.65 \mathrm{mmol} / \mathrm{g}$.

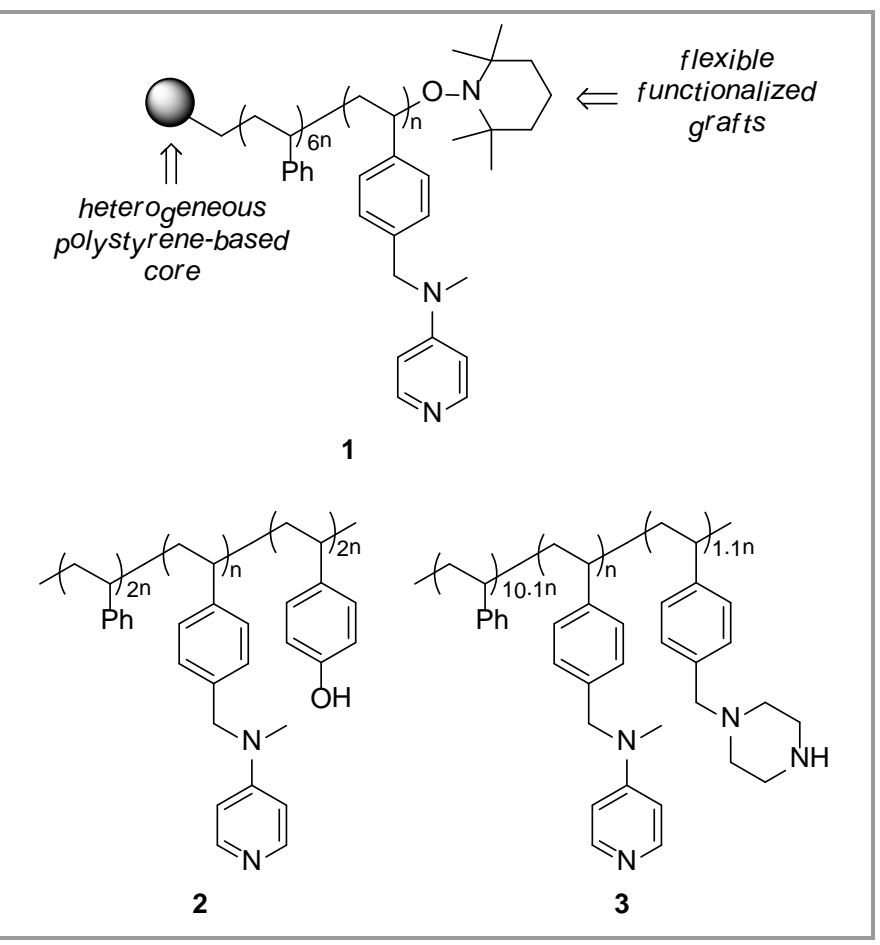

Figure 1 Polymer-supported DMAPs

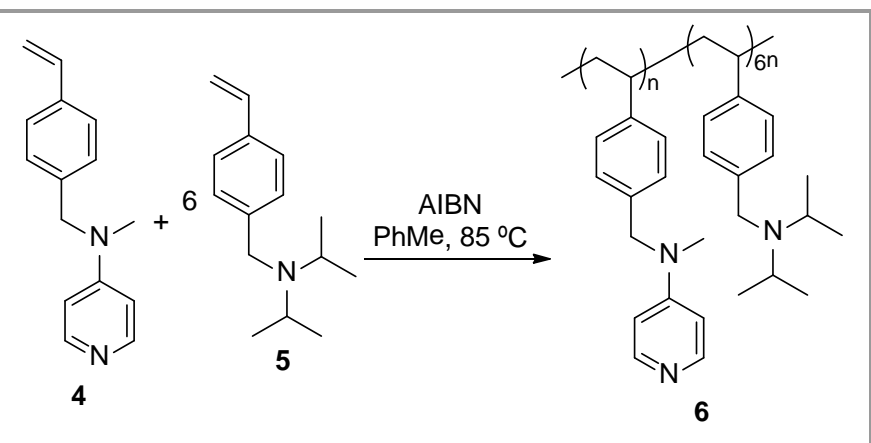

Scheme 1 Synthesis of bifunctional polystyrene 6

With bifunctional polymer $\mathbf{6}$ in hand we examined its utility in the esterification reaction between 1phenylethanol (7A) and excess acetic anhydride (8a) to form ester 9Aa on a $1.5 \mathrm{mmol}$ scale (Table 1 ). Since we envisioned the need for a slight excess of the basic tertiary amine groups, we used 20 mole percent of $\mathbf{6}$ based on DMAP loading, which corresponded to using 1.2 equivalents of the amine groups (entry 1). Gratifyingly, 6 was completely soluble in dry toluene at room temperature, and the reaction was complete after 1 hour. The expected product 9Aa could be isolated essentially pure 
and in quantitative yield after only precipitation of the polymer by-product using hexane (in which $\mathbf{6}$ and its protonated form are insoluble), washing of the reaction mixture with aqueous base and brine, and concentration. We next gradually reduced the amount of catalytic DMAP groups used and found that with 5 or 2 mole percent DMAP (entries 2 and 3), quantitative yield of 9Aa could still be obtained. Only when 1 mole percent of DMAP was used did the reaction not go to completion in 1 hour (entry 4), with only 64\% isolated yield obtained after separation of unreacted starting materials. Significantly when the corresponding homogeneous small molecules DMAP and $i \mathrm{Pr}_{2} \mathrm{EtN}$ were used instead of polymer 6 (20 and 120 mole percent, respectively), 9Aa was isolated in only $73 \%$ yield after 1 hour (entry 5). Thus, it seems that polymer $\mathbf{6}$ is more efficient in esterification reactions than the combination of DMAP and $i \mathrm{Pr}_{2} \mathrm{EtN}$ in toluene at room temperature, and the observation that the reactions are efficient even when less than a full equivalent of tertiary amine is consistent with similar findings reported by Ishihara and coworkers. $^{11}$

Table 1 Esterification Reactions Using $\mathbf{7 A}$ and $\mathbf{8} \mathbf{a}^{\mathrm{a}}$

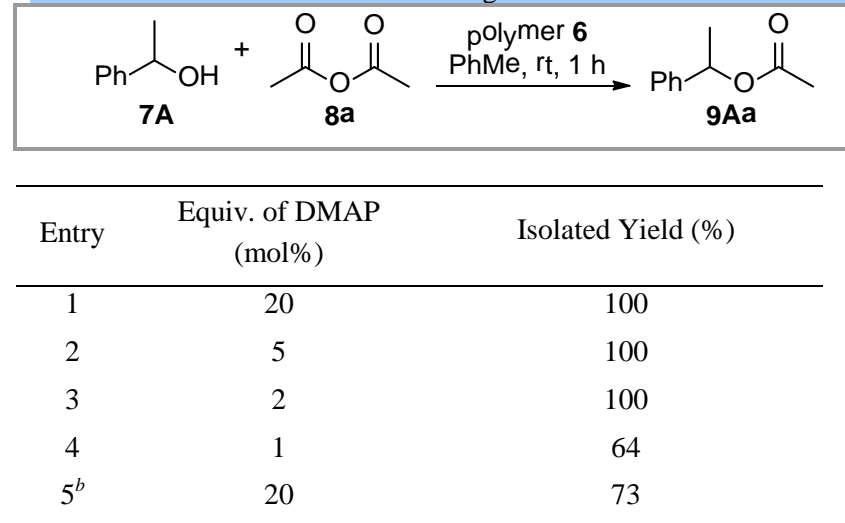

${ }^{\mathrm{a}}$ Reaction conditions: alcohol 4A (1.5 mmol), acid anhydride 5a $(1.8$ mmol), polymer 6 and PhMe (3.0 mL) were stirred at rt for $1 \mathrm{~h}$.

${ }^{\mathrm{b}} \mathrm{DMAP}(0.3 \mathrm{mmol})$ and $i \mathrm{Pr}_{2} \mathrm{EtN}(1.8 \mathrm{mmol})$ were used in place of 6 .

After demonstrating the utility of $\mathbf{6}$ in an esterification reaction, we next sought to study both the substrate scope and the recyclability of the polymer in order to assess its practicality. Thus, we then conducted a series of larger scale acylation reactions (15 mmol scale) using hindered secondary and tertiary alcohols 7A-D and acid anhydrides 8a-c (Figure 2) using the same sample of $\mathbf{6}$ for each successive reaction (Table 2). It should be noted that we chose to use variously substituted benzylic alcohols simply because we wanted to prepare nonvolatile products that could be easily visualized by TLC analysis, and it is assumed that non-benzylic alcohols would work equally well as substrates. After each reaction the polymer was precipitated from the reaction mixture, washed with aqueous $\mathrm{NaOH}$, dried, and weighted prior to use in the next reaction cycle. Initially 9Aa was prepared three successive times using 0.467 grams of 6 at the outset (0.02 equivalents DMAP), with excellent results obtained (entries 1-3). Next, 7B was reacted with 8a three times to form $\mathbf{9 B a}$ with excellent results and no evidence of product contamination from the previous reactions (entries 4-6). This was followed by reaction of more sterically hindered 7C with 8 a to form 9Ca three times in reactions that required 3 hours for completion, but that still afforded quantitative yield (entries 7-9). We then changed the acid anhydride from $\mathbf{8 a}$ to $\mathbf{8 b}$ and $\mathbf{8 c}$, and reactions of these with 7A to form $\mathbf{9 A b}$ and $\mathbf{9 A c}$, respectively, were complete in 1 hour (entries 10-15). While high yields were afforded in every cycle, we scaled down the reactions for entries 13 and 14 due to the gradual loss of $\mathbf{6}$. Finally tertiary alcohol 7D was reacted with $\mathbf{8 a}$ in three reactions that required heating to $100{ }^{\circ} \mathrm{C}$ for 48 hours in order to obtain moderate yieds of 9Da (entries 16-18).

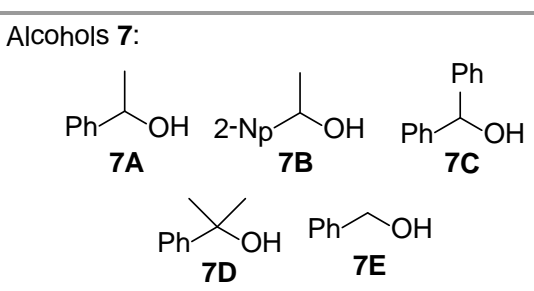

Acylating reagents 8 :<smiles>CCC(=O)OC(=O)CC</smiles><smiles>CC(C)C(=O)OC(=O)C(C)C</smiles>

Products 9:
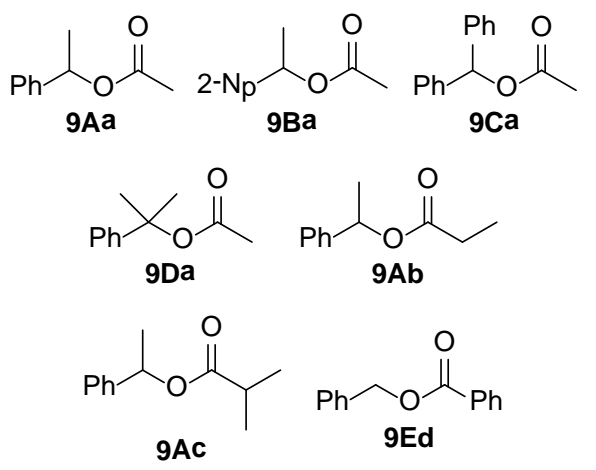

Figure 2 Starting materials and products of esterification reactions

Next we examined the utility of $\mathbf{6}$ in esterification reactions using an acid chloride instead of an acid anhydride. Thus, 7E was reacted with benzoyl chloride (8d) using 6 (0.2 equivalents DMAP) in dry toluene in a reaction that required 6 hours to proceed to completion (Table 3 , entry 1). As before, the polymer was precipitated at the end of the reaction, treated with aqueous $\mathrm{NaOH}$, dried, and reused in the next reaction. Gratifyingly we found $\mathbf{6}$ to be highly recyclable in these reactions as well. (entries 2-5). In all reactions, quantitative yield of pure 9Ed was obtained.

Table 3 Esterification Reactions Using 7E and 8d Mediated by 6 


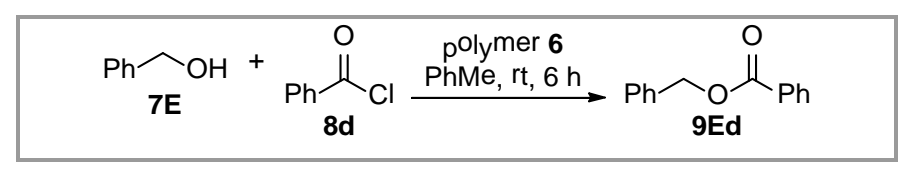

\begin{tabular}{cc}
\hline Reaction Cycle & Isolated Yield (\%) \\
\hline 1 & 100 \\
2 & 100
\end{tabular}

$\begin{array}{ll}3 & 100 \\ 4 & 100 \\ 5 & 100\end{array}$

${ }^{a}$ Reaction conditions: alcohol 4E (0.5 mmol), acid chloride $5 \mathbf{d}$ (0.6 mmol), polymer 6 (0.1 mmol DMAP), and PhMe $(3.0 \mathrm{~mL})$ were stirred at rt for $6 \mathrm{hr}$.

Table 2 Esterification Reactions Using Various Alcohols 7 and Acid Anhydrides 8 Mediated by $\mathbf{6}^{\mathrm{a}}$

\begin{tabular}{|c|c|c|c|c|c|c|}
\hline & & & & $\mathrm{R}^{1}$ & & \\
\hline Reaction Cycle & 7 & 8 & 9 & $\mathrm{~T}(\mathrm{hr})$ & Isolated Yield (\%) & $\begin{array}{c}\text { Mass of Recov- } \\
\text { ered } 6 \text { (mg) }\end{array}$ \\
\hline 1 & 7A & $8 \mathbf{a}$ & 9Aa & 1 & 100 & 461 \\
\hline 2 & 7A & $8 \mathbf{a}$ & 9Aa & 1 & 99 & 451 \\
\hline 3 & 7A & 8a & 9Aa & 1 & 98 & 450 \\
\hline 4 & 7B & $8 \mathbf{a}$ & 9Ba & 1 & 100 & 448 \\
\hline 5 & 7B & $8 \mathbf{a}$ & 9Ba & 1 & 100 & 447 \\
\hline 6 & 7B & $8 \mathbf{a}$ & 9Ba & 1 & 100 & 451 \\
\hline 8 & 7C & $8 \mathbf{a}$ & 9Ca & 3 & 99 & 441 \\
\hline 9 & 7C & $8 \mathbf{a}$ & 9Ca & 3 & 100 & 446 \\
\hline 10 & 7A & $8 b$ & 9Ab & 1 & 100 & 441 \\
\hline 11 & 7A & $8 b$ & 9Ab & 1 & 99 & 440 \\
\hline 12 & 7A & $8 b$ & 9Ab & 1 & 99 & 441 \\
\hline 13 & 7A & 8c & 9Ac & 1 & 100 & 342 \\
\hline 14 & 7A & $8 c$ & 9Ac & 1 & 100 & 340 \\
\hline 15 & 7A & $8 c$ & 9Ac & 1 & 100 & 338 \\
\hline 16 & 7D & $8 a$ & 9Da & 48 & 82 & $215^{\mathrm{b}}$ \\
\hline 17 & 7D & $8 a$ & 9Da & 48 & 74 & $219^{\mathrm{b}}$ \\
\hline 18 & 7D & $8 a$ & 9Da & 48 & 75 & $216^{\mathrm{b}}$ \\
\hline
\end{tabular}

${ }^{\mathrm{a}}$ Reaction conditions: for entries 1-13, alcohol 4 (15 mmol), acid anhydride 5 (18 mmol), polymer 6 and PhMe (30 mL) were stirred at rt for the indicated time; for entries 14-15, alcohol 4 (10 mmol), acid anhydride 5c (12 mmol), polymer 6 and PhMe (20 mL) were stirred at rt for 1 h; for entries 16-18, alcohol 4D (10 mmol), acid anhydride 5a (12 mmol), polymer 6 and PhMe (20 mL) were stirred at $100{ }^{\circ} \mathrm{C} 48 \mathrm{~h}$.

${ }^{\mathrm{b}}$ Reaction was not complete and 9Da was purified by column chromatography.

In summary, we have developed bifunctional linear polystyrene 6, bearing both DMAP and tertiary amine groups, which can be used effectively in esterification reactions between an alcohol and an acid anhydride or an acid chloride. The macromolecular nature of $\mathbf{6}$ allows it to be readily separated from the ester products, and thus chromatographic purification is not necessary. Additionally, $\mathbf{6}$ is highly recyclable, with no apparent decrease in activity after numerous reaction cycles, and it can be recovered in a highly pure state so that no product contamination is observed when starting materials are changed. Furthermore, it appears that $\mathbf{6}$ is more efficient in esterification reactions that an equivalent mixture of DMAP and $i \mathrm{Pr}_{2} \mathrm{EtN}$ under identical reaction conditions, and this may be due to the polar microenvironment created by the densely functionalized polymer. Studies to determine the generality and origin of this increased efficiency, as well as apply $\mathbf{6}$ and heterogeneous analo- gues of it to other reactions, are currently underway, and the results of these efforts will be reported in due course.

\section{Acknowledgement}

This research was supported financially by the University of Hong Kong and the Research Grants Council of the Hong Kong S. A. R., P. R. of China (Project No. HKU 705510P).

\section{Supporting Information}

Supporting information for this article is available online at http://

\section{References and Notes}

(1) Höfle, G;; Steglich, W.; Vorbrüggen, H. Angew. Chem. Int. Ed. Engl. 1978, 17, 569. 
(2) For selected recent examples, see: (a) Cotanda, P.; Lu, A.; Patterson, J. P.; Petzetakis, N.; O'Reily, R. K. Macromolecules 2012, 45, 2377. (b) Khamatnurova, T. V.; Johnson, M.; Santana, D.; Bazzi, H. S.; Bergbreiber, D. E. Top Catal. 2014, 57, 1438. (c) Zhen, Y.; Lin, H.; Wang, S.; Tao, M. RSC Adv. 2014, 4, 26122. (d) Okuno, Y.; Isomura, S.; Sugamata, A.; Tamahori, K.; Fukuhara, A.; Kashiwagi, M.; Kitagawa, Y.; Kasai, E.; Takeda, K. ChemSusChem 2015, 8, 1629.

(3) For a very recent report regarding the use of a porous solid polymeric acid catalyst in esterification reactions between carboxylic acids and alcohols, see: Kim, Y.-H.; Han, J.; Jung, B. Y.; Baek, H.; Yamada, Y. M. A.; Uozumi, Y.; Lee, Y.-S. Synlett 2016, 27, 29.

(4) Lu, J.; Toy, P. H. Synlett 2011, 22, 659.

(5) (a) Kwong, C. K.-W.; Huang, R.; Zhang, M.; Shi, M.; Toy, P. H. Chem. Eur. J. 2007, 13, 2369. (b) Kwong, C. K.-W.; Fu, M. Y.; Law, H. C.-H.; Toy, P. H. Synlett 2010, 21, 2617.

(6) Lu, J.; Toy, P. H. Synlett 2011, 22, 1723.

(7) For reports regarding DMAP attached to basic polyethyleneimines in ester hydrolysis reactions, see: (a) Hierl, M. A.; Gamson, E. P.; Klotz, I. M. J. Am. Soc. 1979, 101, 6020. (b) Delaney, E. J.; Wood, L. E.; Klotz, I. M. J. Am. Chem. Soc.1982, 104, 799.

(8) (a) Leung, P. S.-W.; Teng, Y.; Toy, P. H. Org. Lett. 2010, 12, 4996. (b) Lu, J.; Toy, P. H. Synlett 2011, 22, 3085. (c) Teng, Y.; Lu, J.; Toy, P. H. Chem. Asian J. 2012, 7, 351. (d) Lu, J.; Toy, P. H. Pure Appl. Chem. 2013, 85, 543. (e) Xia, X.; Toy, P. H. Beilstein J. Org. Chem. 2014, 10, 1397. (f) Derible, A.; Yang, Y.-C.; Toy, P. H.; Becht, J.-M.; Le Drian, C. Tetrahedron Lett. 2014, 55, 4331. (g) Xia, X.; Toy, P. H. Synlett 2015, 26, 1737.

(9) See Supporting Information for details.

(10) General Procedure for Esterification Reactions: Acylating reagent 7 (1.2 equiv.) was slowly added dropwise into a stirred solution of alcohol 8 (1.0 equiv.) and polymer $\mathbf{6}$ in dry toluene $(30 \mathrm{~mL})$. The reaction mixture was stirred at room temperature, and when TLC indicated that 8 had been completely consumed, the reaction mixture was slowly added dropwise into hexane $(150 \mathrm{~mL})$. The resulting precipitate was filtered off and the filtrate was washed with saturated aqueous $\mathrm{Na}_{2} \mathrm{CO}_{3}(50 \mathrm{~mL})$. The organic layer was washed with brine $(50 \mathrm{~mL})$ and dried over anhydrous magnesium sulfate, and concentrated under reduced pressure to afford product ester 9 in an essentially pure state according to ${ }^{1} \mathrm{H}$ NMR analysis. The collected precipitate was dissolved methanol (8 $\mathrm{mL}$ ), and was slowly added dropwise to a vigorously stirred solution of $0.1 \mathrm{~N}$ sodium hydroxide dissolved in methanol $(100 \mathrm{~mL})$ at $0{ }^{\circ} \mathrm{C}$. The resulting light yellow precipitate was filtered, washed with methanol (40 mL), and dried under reduced pressure for reuse in the next reaction.

1-Phenylethyl acetate (9Aa): ${ }^{1} \mathrm{H}$ NMR $\left(400 \mathrm{MHz}, \mathrm{CDCl}_{3}\right) \delta$ ppm 1.49 (d, 3H, $J=6.8 \mathrm{~Hz}), 1.98(\mathrm{~s}, 3 \mathrm{H}), 5.86(\mathrm{q}, 1 \mathrm{H}, J=$ 6.8 Hz), 7.22-7.33 (m, 5H). ${ }^{13} \mathrm{C} \mathrm{NMR}\left(100 \mathrm{MHz} \mathrm{CDCl}_{3}\right) \delta$ ppm 21.0. 22.0, 72.0, 125.9 (2C), 127.6, 128.3 (2C), 169.8. LRMS for $\mathrm{C}_{10} \mathrm{H}_{12} \mathrm{O}_{2}$ : calc 164.1, found 164.1.

(11) Sakakura, A.; Kawajiri, K.; Ohkubo, T.; Kosugi, Y.; Ishihara, K. J. Am. Chem. Soc. 2007, 129, 14775. 
Graphical abstract

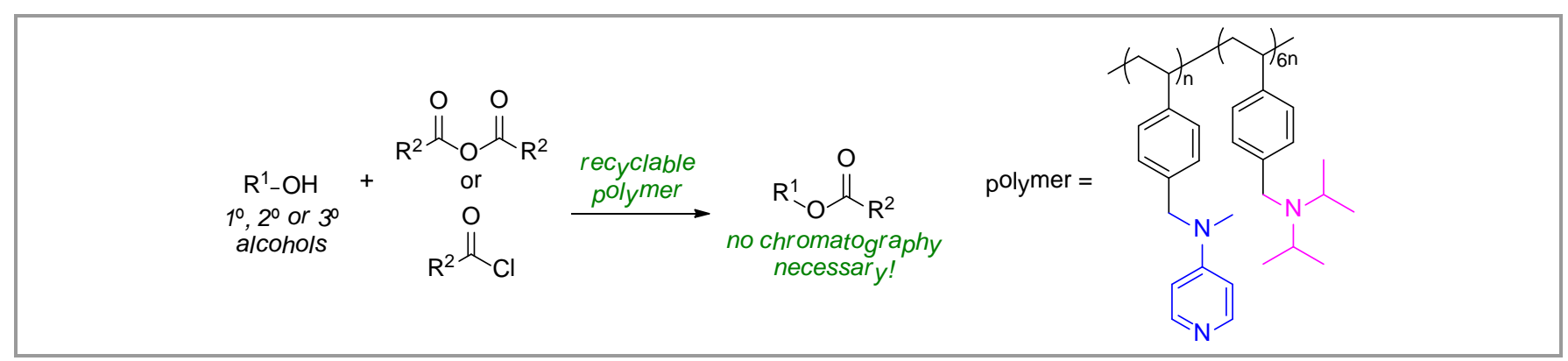

